\title{
(C) OPEN ACCESS \\ Sedentary time in older men and women: an international consensus statement and research priorities
}

\author{
Shilpa Dogra, ${ }^{1}$ Maureen C Ashe, ${ }^{2}$ Stuart J H Biddle, ${ }^{3,4}$ Wendy J Brown, ${ }^{5}$ \\ Matthew P Buman, ${ }^{6}$ Sebastien Chastin, ${ }^{7,8}$ Paul A Gardiner, ${ }^{5}$ Shigeru Inoue, ${ }^{9}$ \\ Barbara J Jefferis, ${ }^{10}$ Koichiro Oka, ${ }^{11}$ Neville Owen, ${ }^{12,13}$ Luís B Sardinha, ${ }^{14}$ \\ Dawn A Skelton, ${ }^{7}$ Takemi Sugiyama, ${ }_{15}^{15}$ Jennifer L Copeland ${ }^{16}$
}

For numbered affiliations see end of article.

\section{Correspondence to}

Dr Shilpa Dogra, Faculty of

Health Sciences (Kinesiology), University of Ontario Institute of Technology, Oshawa L1H 7K4, Canada; shilpa.dogra@uoit.ca

Accepted 28 June 2017

\section{Linked}

http://dx.doi.org/10.1136/ bjsports-2016-097210

CrossMark

\section{To cite: Dogra $S$}

Ashe MC, Biddle SJH,

et al. Br J Sports Med

2017:51:1526-1532.

This consensus statement has been endorsed by the following societies: Academy of Geriatric Physical Therapy, Exercise \& Sports Science Australia, Canadian Centre for Activity and Aging, Society of Behavioral Medicine, and the National Centre for Sport and Exercise Medicine.

\section{ABSTRACT}

Sedentary time is a modifiable determinant of poor health, and in older adults, reducing sedentary time may be an important first step in adopting and maintaining a more active lifestyle. The primary purpose of this consensus statement is to provide an integrated perspective on current knowledge and expert opinion pertaining to sedentary behaviour in older adults on the topics of measurement, associations with health outcomes, and interventions. A secondary yet equally important purpose is to suggest priorities for future research and knowledge translation based on gaps identified. A five-step Delphi consensus process was used. Experts in the area of sedentary behaviour and older adults ( $n=15)$ participated in three surveys, an in-person consensus meeting, and a validation process. The surveys specifically probed measurement, health outcomes, interventions, and research priorities. The meeting was informed by a literature review and conference symposium, and it was used to create statements on each of the areas addressed in this document. Knowledge users $(n=3)$ also participated in the consensus meeting. Statements were then sent to the experts for validation. It was agreed that selfreport tools need to be developed for understanding the context in which sedentary time is accumulated. For health outcomes, it was agreed that the focus of sedentary time research in older adults needs to include geriatric-relevant health outcomes, that there is insufficient evidence to quantify the dose-response relationship, that there is a lack of evidence on sedentary time from older adults in assisted facilities, and that evidence on the association between sedentary time and sleep is lacking. For interventions, research is needed to assess the impact that reducing sedentary time, or breaking up prolonged bouts of sedentary time has on geriatric-relevant health outcomes. Research priorities listed for each of these areas should be considered by researchers and funding agencies.

\section{INTRODUCTION}

Sedentary (sitting) time is an important modifiable determinant of health, ${ }^{1}$ and it is an important predictor of healthy ageing. ${ }^{2}$ While research on the causes and consequences of excessive sedentary time is expanding rapidly in all populations, this line of inquiry should be of particular interest to researchers, gerontologists and healthcare practitioners working with older adults. Research from over 10 countries indicates that older adults are sedentary for approximately 10 hours per day. ${ }^{3}$ The volume of sedentary time that older adults accumulate is greater than any other age group ${ }^{4-9}$; however, even among older adults, older age is associated with more sedentary time. ${ }^{10}$ While there is no consistent sex difference in total sedentary time, there appear to be differences in the patterns and types of sedentary behaviours in which older men and women engage. ${ }^{11-13}$

Managing sedentary time is important for the prevention of disease and disability ${ }^{14-16}$ and also for the management of existing conditions. ${ }^{17} 18$ Too much sitting may have particularly deleterious consequences for older adults, in part because they accumulate the least physical activity of any age group. ${ }^{4}$ Very few older adults are engaging in the relatively high volume of daily moderate-to-vigorous intensity physical activity that appears to be necessary to counteract the adverse effects of too much sitting ${ }^{19}$ and may have neither the physical capacity nor the desire to achieve such a goal. ${ }^{20-23}$

Ageing is associated with a decline in physical function; prolonged periods of muscular unloading may exacerbate losses in muscle and bone mass and further increase the risk of falls, frailty and dependence. The unique challenges facing older adults, such as age-associated functional (physical and cognitive) changes, may be just as important as conventional morbidity and mortality outcomes, as they have been shown to more strongly impact the quality of life of older adults. ${ }^{24-26}$ These issues, combined with the ubiquitous nature of sedentary behaviour, means that understanding the relationship between sedentary time and healthy ageing is of particular importance. This is even more urgent given that globally there will be more than two billion older adults by the year $2050 .^{27}$ 
Considerable research has been conducted to better understand the determinants of sedentary time among older adults. A transdisciplinary, international consensus framework that includes research priorities pertaining to determinants of sedentary time was recently created. ${ }^{28}$ Through the review process, unique determinants such as physical function, living arrangements and the presence of chronic disease were identified for older adults, ${ }^{29}$ further highlighting the need for age-specific research in this area. To further advance this age-specific research, perspective is needed on measurement techniques and the current state of knowledge on the impact of sedentary time on healthy ageing.

This consensus statement was generated by 15 researchers from five countries across four continents. All group members were researchers in the area of sedentary time among older adults, with expertise in physiology, psychology/behaviour change, public health, rehabilitation, measurement of sedentary time and/or epidemiology. The purpose of this statement is, first, to provide an integrated perspective on current knowledge and expert opinion on three main issues as they relate to older men and women: (1) the validity/reliability of self-reported sedentary time measures compared with objectively measured data; (2) existing knowledge of health consequences associated with sedentary time, including dose response; and (3) interventions that show promise for reducing sedentary time. To advance the study of sedentary time and healthy ageing at a pace that reflects the demands of rapid population ageing, research priorities must be established. Thus, a second purpose was to identify 5 -year research priorities for the field and provide information that is relevant for practitioners and other knowledge users concerned with improving the health of older men and women.

\section{METHODS}

\section{Terminology}

For this statement, older adults were those aged 60 years and older. ${ }^{30}$ However, it should be noted that older adults have a range of functional abilities that do not necessarily correlate with chronological age. Therefore, older adults across the mobility spectrum and, those living in different settings (community-dwelling vs various levels of assisted living) were considered.

Throughout this consensus statement, we use the term 'sedentary time' to describe time spent in a seated or reclining posture with low energy expenditure. This is a widely accepted definition of sedentary time ${ }^{31}$; however, it is important to note that much of the available research evidence is not, as yet, derived from the use of measurement tools that can explicitly identify posture or behaviours during sedentary time. Sedentary time is a multifaceted construct. There are many different types of sedentary behaviours (eg, watching TV, reading or transportation), and they can occur in different domains or settings (eg, home or work), at different times of day, alone or with others. ${ }^{32}$ The context of sedentary time is an important consideration for research on health outcomes and the development of interventions.

\section{Process}

This consensus statement was created using a standard Delphi consensus process that was led by SD and JLC. ${ }^{33} 34$ In accordance with recommendations, experts in the field of sedentary time and ageing were identified through PubMed searches and through recommendations from previously identified experts. Twenty-six experts were initially sent an invitation to participate in the consensus process. Of these, 13 experts agreed to participate (in addition to SD and JLC). All these experts regularly publish in the area and appropriately represent a breadth of perspectives on the topics included in this statement.

The Delphi process consisted of three surveys, an in-person consensus meeting, and expert validation. Step one consisted of a survey of six open-ended questions related to each of the areas of interest. They were stated as follows:

- What is the validity/reliability of self-reported sedentary time compared with objectively measured sedentary time in older men and women?

- What is the range of health consequences and the dose of sedentary time associated with these consequences in older men and women?

-What intervention strategies have shown promise in reducing sedentary time among older men and women?

- What are the important age, sex (biological) and gender (sociocultural) differences in sedentary time?

- What are the priorities for future research?

- What are the priorities for knowledge translation and what are the key messages we can disseminate at this time?

The question pertaining to sex and gender was specifically included due to growing concerns over the inappropriate use of these terms in health research and their impact on the interpretation of findings. ${ }^{356} \mathrm{Sex}$ is a set of biological attributes (physical and physiological) in humans, while gender refers to social constructs that cannot be defined using binary terms. ${ }^{37}$

Data from step one were qualitatively analysed and categorised into themes that then informed the development of the second survey. In step two, a survey with a series of statements on the same issues were presented using Likert scales and statements for ranking. Results from step two were used to inform the next survey. In step three, the survey used Likert scales to determine agreement on statements pertaining to sedentary time patterns, and age, sex, and gender differences. They were also used for prioritising the need for individual and population level interventions, and research priorities for cross-sectional, longitudinal, randomised controlled, qualitative, and laboratory/mechanistic studies.

Prior to the consensus meeting (step four), a review of the literature on sedentary time and older adults was synthesised and sent to the experts with the results of the three surveys. The literature review was sent to the experts after the three surveys were conducted to ensure that both the experts' perspectives and the evidence could be discussed at the meeting. The experts and knowledge users also attended a 90 min symposium at the World Congress on Active Aging on 30 June 2016. The symposium summarised the main findings of the review, as well as issues pertaining to sex, gender, and age differences in sedentary time and health outcomes, determinants of sedentary time, and patterns of sedentary time. Eleven of the experts (four were unable to attend but were sent audiotapes and notes on the meeting afterwards) as well as three knowledge users met on 2 July 2016 in Melbourne, Australia. The purpose of the meeting was to discuss results of the surveys in the context of the areas of interest and in light of the literature review. ${ }^{38}$ The goal was to create initial statements in each area as well as to identify the most important priorities for both research and knowledge translation.

After the meeting, consensus statements were categorised according to level of evidence (table 1) and sent to the expert group for review and validation (step five). This was to ensure that the statements provided below reflect the views of the experts who participated in the surveys and meeting. 


\begin{tabular}{|c|c|c|}
\hline Category & Methodology & Descriptor \\
\hline A & Randomised, controlled trials (RCTs) & Data are overwhelmingly from RCT studies, and findings are consistent. \\
\hline C & Non-RCTs, observational studies & $\begin{array}{l}\text { Outcomes are from uncontrolled, non-randomised and/or observational studies (overwhelmingly } \\
\text { cross-sectional). }\end{array}$ \\
\hline
\end{tabular}

\section{FINDINGS}

The findings of the consensus process are presented by research question. Consensus statements are listed following a summary of the literature review and the survey results (steps one to three) for each question. Please see the accompanying review in this issue for further details on the literature pertaining to measurement, associations with health outcomes, and interventions. ${ }^{38}$

\section{What is the validity/reliability of self-reported sedentary time compared with objectively measured data in older men and women?}

The validity/reliability of self-reported sedentary time is an important issue as it influences the interpretation of studies using self-report tools. From the nine studies reviewed in the literature, seven studies compared self-report tools with accelerometers, ${ }^{39-45}$ and two compared self-report tools with inclinometers. ${ }^{46} 47$ Since inclinometers provide information on posture, and the definition of sedentary behaviour includes reference to posture, self-report tools should be validated against inclinometers. All but two studies reported that the self-reported tool being assessed underestimated total sedentary time. One tool was found to overestimate total sedentary time among those who accumulated more than $640 \mathrm{~min} / \mathrm{day}^{39}$, while another tool was found to overestimate sedentary time by 1 hour/day. ${ }^{46}$ The testretest reliability of specific sedentary activities, particularly TV time and computer use, was found to be acceptable. ${ }^{39}{ }^{40}$ Further information and interpretation pertaining to these studies can be found in the accompanying review. ${ }^{38}$

There was consensus that self-report measures of sedentary time have limitations in terms of validity and reliability, and that improvements in these tools are needed. However, it was acknowledged that self-report is important for examining the context of sedentary time and identifying specific types of sedentary behaviours (eg, TV viewing, reading and driving). In light of the value that self-report tools can add to sedentary time research, using valid and reliable methods of studying, the context of sedentary time was identified as a priority. The issue of measurement of movement behaviours, including sleep, over a 24-period was discussed at the meeting. Sleep was not raised through any of the three prior surveys, nor was it mentioned in any of the studies on self-report.

The consensus meeting, review of evidence and expert validation led to the development of the following statements on the use of self-reported sedentary time for research with older men and women:

1. Self-report has limited value for the measurement of total sedentary time. (Evidence category: $C$ and D)

2. The context (what, when, where and with whom) of sedentary time is important and, at present, is primarily assessed using self-report tools. (Evidence category: D)

3. A comprehensive approach that includes subjective and objective measures that account for behaviours across the
24 hours, such as sleep, posture, active behaviours, and sedentary time, is needed. (Evidence category: D)

\section{What is the range of health consequences and the dose of sedentary time associated with these consequences in older men and women?}

Research on sedentary time and health outcomes in older adults has focused primarily on outcomes of mortality and cardiometabolic disease risk factors or outcomes. A growing body of longitudinal evidence suggests an association between sedentary time and mortality. ${ }^{48} 49$ There also appears to be an association between sedentary time and some cardiometabolic disease risk factors, although much of the evidence comes from cross-sectional studies. ${ }^{50-52}$ There is a dearth of research on the association of sedentary time with other health outcomes such as cancer, pulmonary disease, mental health and more. Furthermore, few high-quality studies have looked at the association between sedentary time and geriatric-relevant health outcomes, that is, non-disease outcomes that strongly influence quality of life in this population.

Geriatric-relevant outcomes include physical function, cognitive function, urinary incontinence and mental health (depressive symptoms). Research on these outcomes and their association with sedentary time is covered extensively in the accompanying review. $^{38}$ Briefly, evidence from cross-sectional studies indicates that there is an association between sedentary time and performance on functional fitness tests, strength, activities of daily living, and falls; there is also evidence of an association between breaks in sedentary time and physical function. Sex and age differences have been reported for some of these associations. For cognitive function, evidence from cross-sectional studies suggests that there is an association between sedentary time and dementia/mild cognitive impairment, performance on neurocognitive tests and brain structure/activity. However, this research should be interpreted with caution as many studies used self-report and did not define a dose of sedentary time. Only one study to date has assessed the association between sedentary time and urinary incontinence; no association was found. Evidence from a small number of cross-sectional studies of mental health suggests that higher sedentary time may be associated with greater psychological distress and depressive symptoms. To date, studies on sedentary time and sleep show no association. Finally, sedentary time appears to be inversely associated with general well-being and quality of life, based on a small number of cross-sectional studies. Of note, the majority of studies reviewed on the association between geriatric-relevant outcomes and sedentary time were cross-sectional, thus, reverse causality cannot yet be ruled out, and the term 'consequences' cannot be appropriately applied to this work. Although our objective was to assess health consequences, at this time, we can only assess associations with health outcomes. 
Many studies reviewed found that associations between sedentary time and health outcomes remained significant after adjusting the models for moderate to vigorous intensity physical activity. There is also research to suggest that among young and middle-aged adults, accumulating $60-75$ min of moderate to vigorous intensity physical activity can negate the effects of prolonged sitting ${ }^{19}$; no such data are available on older adults at this time. Importantly, few older adults meet minimum guidelines for physical activity. ${ }^{53}$ Therefore, reducing sedentary time may have greater practical implications for older adults than it would for younger adults. More work is needed to elucidate the interactive and independent effects of sedentary time and physical activity on the health of older adults.

Through the first three steps of the Delphi process, the effect of sedentary time on physical function and cognitive function were identified as key areas that require more research, as were mental health, social isolation, and quality of life. It was generally agreed that there is a graded relationship between sedentary time and health but that this relationship likely differs by health outcome and likely is not linear. It was also agreed that due to lack of evidence at this time, it is difficult to identify or quantify a threshold where risk would become clinically relevant. The context in which sedentary time is accumulated is important to health outcomes; however, the mechanism for these contextual differences is not fully understood. The dose of sedentary time associated with negative health outcomes may vary depending on the type of sedentary behaviour; that is, activities that are cognitively and socially engaging may have different effects than those that are less engaging. ${ }^{12}$

Throughout the surveys, there was general consensus that it is important to consider age when assessing physical function, cognitive function, isolation/loneliness, cardiometabolic disease, and quality of life; however, during the in-person meeting, it was agreed that functional ability may be more important than chronological age. Poor functional ability may be a determinant as well as a consequence of sedentary behaviour and physical inactivity, and it may affect the context of sedentary time. Specifically, older adults may live independently or in assisted living facilities. Little research has been done to understand the unique impact of these environments on sedentary time accumulation. The experts also discussed the need for research in hospital settings. In many countries, older adults are admitted to the hospital for non-life threatening conditions. This may be associated with extended in-hospital stays that lead to an increase in sedentary time and physical inactivity. ${ }^{54}$

It was also agreed that sex may influence the relationship between sedentary time and specific health outcomes, such as physical function, ${ }^{5556}$ metabolic syndrome,${ }^{52}$ mental health ${ }^{57}$ and quality of life. ${ }^{2}$ To our knowledge, no studies have specifically addressed gender differences in the area of sedentary behaviour and ageing research. However, researchers have suggested that social and cultural norms about social behaviour are distinctly different, and thus examining gender is important in this field. ${ }^{57}$

The consensus meeting, review of evidence and expert validation led to the development of the following statements on the association between sedentary time and health outcomes in older men and women:

1. There are associations of sedentary time with all-cause mortality, obesity, cardiometabolic disease, and physical function (Evidence category: C). There is limited evidence on the association of sedentary time with well-being, cancer, pulmonary disease, stroke as well as with geriatricrelevant outcomes including mobility, cognitive function, incontinence, and mental health. (Evidence category: D)
2. To date, there is cross-sectional evidence that suggests a graded relationship of sedentary time with health. The available evidence is insufficient to quantify the threshold associated with clinically relevant risk. (Evidence category: D)

3. Associations between sedentary time and health outcomes have mainly been studied in community-dwelling older adults; more research is needed in different settings such as hospitals and assisted living facilities. (Evidence category: D)

4. Nocturnal and daytime sleep duration and quality may play important roles in the associations of sedentary time and health. Better methods to distinguish sleep from sedentary time are needed in order to fully understand these roles. (Evidence category: D)

5. The interaction between sedentary time and physical activity is important. Interactions between sedentary time and physical activity should be tested in all studies. (Evidence category: C and D)

6. Other variables such as sex, functional capacity, and setting may influence the relationship between sedentary time and health, but these have not been well studied. They should be included in future work; researchers are also encouraged to reanalyse previous work considering these important variables. (Evidence category: C and D)

7. Patterns of sedentary time are associated with some health outcomes; longer bouts and fewer breaks in sedentary time appear to be associated with worse health regardless of total sedentary time. Longitudinal and experimental research is needed to understand the impact of these patterns on specific health outcomes. (Evidence category: C and D)

\section{What interventions have shown promise in reducing sedentary time among older men and women?}

High-quality intervention research on reducing sedentary time in older adults is sparse. Of the 13 studies available, seven specifically targeted reducing sedentary time ${ }^{58-64}$ while the remaining either included or exclusively targeted increasing physical activity. ${ }^{63}$ 65-69 Only six were randomised controlled trials, ${ }^{66-70}$ and only five had follow-up periods of 6 months or longer. ${ }^{66-70}$ Most studies found statistically significant decreases in total sedentary time as a result of the intervention, and some also reported a decrease in prolonged sedentary time, or a reduction in specific types of sedentary activities. Most intervention studies did not examine changes in health outcomes; therefore, at this time, we cannot evaluate the clinical impact of reducing sedentary time in older adults. ${ }^{63}{ }^{64}{ }^{66}$ Further information and interpretation pertaining to these interventions can be found in the supplementary table and the intervention section of the accompanying review. ${ }^{38}$

Through the first three steps of the Delphi process, interventions targeting the environment as well as individual behaviour change were both considered important. In addition to reducing total sedentary time, breaking up sedentary time was identified as an important strategy. There was no consensus on whether interventions that focus on physical activity will be effective for reducing sedentary time, or if interventions should focus on sedentary time specifically. It was noted that interventions with a social component and interventions that were accessible to older adults across the mobility spectrum would be important considerations.

The consensus meeting, review of evidence and expert validation led to the development of the following statements on sedentary time interventions targeting older men and women: 
1. A better understanding of the correlates and determinants of different types of sedentary time can be used to inform the development of interventions. (Evidence category: D)

2. Individual behaviour change interventions show promise for reducing sedentary time in the short term (Evidence category: B); long-term changes and environmental or organisational level interventions have not been assessed.

3. Intervention research should focus on older adults across the spectrum of function from community-dwelling older adults to those in assisted living environments. (Evidence category: D)

4. Intervention studies should measure changes in behaviour as well as changes in health outcomes. Without measuring both, clinically meaningful changes in sedentary time cannot be established. (Evidence category: D)

5. Interventions should distinguish between reclining and sitting postures as these may have different implications for people with mobility limitations as well as differential effects on health outcomes. (Evidence category: D)

\section{What are the priorities for knowledge translation and the key messages we can disseminate at this time?}

Three knowledge users from three different countries were invited to the consensus meeting to consult on key knowledge translation priorities. It was agreed that there is a need for evidence-informed messages and guidelines about sedentary time for older adults. However, some research indicates that there is a lack of awareness of current physical activity recommendations ${ }^{71}$ thus innovative methods for disseminating public health messages, particularly among older adults, are also needed.

The following two priorities were identified:

1. Recommendations are needed that integrate messages on movement across the spectrum, from sleep to vigorous intensity physical activity, throughout the 24-hour day.

2. Effective implementation strategies are needed to ensure appropriate uptake of recommendations.

It was also agreed that at this time, knowledge users working with older adults can provide a message that, regardless of age, ability, working status, and home setting, older adults should strive to reduce total sedentary time, break up prolonged periods of sedentary time, and move more. This message is already being disseminated to older adults living in some countries. ${ }^{72}$ Reducing sedentary time should be considered in leisure time, in the home, at work and during transportation.

\section{CONCLUSIONS}

This consensus statement was developed through a five-step Delphi consensus process with an international group of experts in sedentary behaviour in older adults. The process was informed by a review of literature and a conference symposium. The goal was to provide consensus, based on current knowledge and expert opinion, and to help advance the field by identifying key research priorities. First, while self-report methods for measuring sedentary time have value for examining the context of sedentary behaviour, they require improvement from the perspective of validity and reliability. Novel methods of objectively assessing both the dose and context of sedentary time should be explored; these will be important for discerning the health consequences of different types of sedentary behaviours accumulated in different contexts. Second, research on health outcomes needs to expand to include the effect of sedentary time on geriatric-relevant health outcomes of physical and cognitive function, urinary incontinence, depressive symptoms, and overall quality of life.
What are the priorities for future research?

Several important gaps in the field became evident through the literature review and the Delphi process; priorities for research in the next 5 years were identified. The key priorities that emerged were: intervention strategies for reducing sedentary time, determining the dose of sedentary time associated with increased health risk, and determining the health effects of different types of sedentary behaviours (eg, TV vs computer vs reading). It was also noted that it is important for future research to examine sedentary time as a determinant of poor health and as a consequence of poor health. In step three of the Delphi process, research priorities were categorised by study design and health outcome.

The following statements on 5-year research priorities were agreed on at the consensus meeting and through expert validation:

1. New objective methods of providing context for sedentary time (eg, wearable cameras, global positioning system and Wi-Fi) should be further validated, and development of new self-report tools should focus on providing context to devicebased measures.

2. Research on sedentary time is needed in different settings and across the mobility spectrum.

3. Research on sedentary time needs to include geriatricrelevant health outcomes. There is a clear need for longitudinal, mechanistic, and experimental studies specifically looking at physical and cognitive function.

4. Research assessing the effectiveness of sedentary time reduction interventions that target the environment and organisation level, and interventions that compare the effectiveness of physical activity and sedentary time interventions, are needed.

5. Research is needed to develop effective implementation strategies for dissemination of sedentary time messages to older adults and professionals working with older adults.

6. Sex and gender may interact with sedentary time to influence health outcomes. To date, sedentary time research has not appropriately distinguished between sex and gender. Sex and Gender Equity in Research guidelines were developed by an international panel to promote the systematic reporting of sex and gender in research. ${ }^{37}$ Future work should stratify analyses by sex or gender, with careful consideration of the definitions of these terms; a growing number of agencies and peer-reviewed journals require such analyses.

Furthermore, the dose-response relationship between sedentary time and health among older adults must be defined, and the interaction with physical activity should be examined. Third, limited work has studied the effectiveness of various intervention strategies, and this is a key research priority. Fourth, there is a need to provide evidence-informed messages for both practitioners and the public about the effects of sedentary time on the health of older adults. While it is acknowledged that recommendations are needed that integrate movement throughout the entire day, there are gaps in the research that must be addressed before that message is ready for translation. In the meantime, practitioners working with older adults can safely provide a message that all older adults should strive to reduce total sedentary time, break up prolonged periods of sedentary time, and move more. 
Increasing age is associated with a decrease in physical and cognitive function, and sedentary behaviour may be a modifiable risk factor that can reduce the risk of disease and disability among older adults. In light of the ageing population, there is considerable potential for reducing sedentary behaviour to improve quality of life of older adults, and perhaps ease the burden on the healthcare system. We encourage researchers and funding agencies to consider the research priorities listed for each of these areas.

\author{
Author affiliations \\ 'University of Ontario Institute of Technology, Oshawa, Ontario, Canada \\ 2University of British Columbia, Vancouver, British Columbia, Canada \\ ${ }^{3}$ Victoria University, Melbourne, Australia \\ ${ }^{4}$ University of Southern Queensland, Springfield, Australia \\ ${ }^{5}$ University of Queensland, Brisbane, Australia \\ ${ }^{6}$ Arizona State University, Phoenix, Arizona, USA \\ ${ }^{7}$ Glasgow Caledonian University, Glasgow, UK \\ ${ }^{8}$ Ghent University, Ghent, Belgium \\ ${ }^{9}$ Waseda University, Saitama, Japan \\ ${ }^{10}$ University College London, London, UK \\ ${ }^{11}$ Waseda University, Tokyo, Japan \\ ${ }^{12}$ Baker Heart and Diabetes Institute, Melbourne, Australia \\ ${ }^{13}$ Swinburne University of Technology, Melbourne, Australia \\ ${ }^{14}$ CIPER, Faculdade de Motricidade Humana, Universidade de Lisboa, Lisboa, \\ Portugal \\ ${ }^{15}$ Australian Catholic University, Melbourne, Australia \\ ${ }^{16}$ University of Lethbridge, Lethbridge, Canada
}

Correction notice This paper has been amended since it was published Online First. A statement has been added on the first page before the Abstract.

Acknowledgements We would like to thank Bridget Lett from the Canadian Society for Exercise Physiology; Robert (Bob) Laventure from the British Heart Foundation National Centre for Physical Activity and Health, Loughborough University (UK); and Dr Tim Henwood, Principal at AgeFIT Solutions, Australia, for their valuable contributions as knowledge users during the consensus meeting.

Collaborators Bridget Lett, Tim Henwood, and Robert (Bob) Laventure.

Contributors SD and JLC take overall responsibility for the original concept and content. MCA, SJHB, WJB, MPB, SC, PAG, SI, BJJ, KO, NO, LBS, DAS and TS contributed to the content and provided expert review of the article.

Funding This work was supported by the Canadian Institutes of Health Research, and by the Canadian Society for Exercise Physiology.

Competing interests None declared.

Provenance and peer review Not commissioned; externally peer reviewed.

Open Access This is an Open Access article distributed in accordance with the Creative Commons Attribution Non Commercial (CC BY-NC 4.0) license, which permits others to distribute, remix, adapt, build upon this work non-commercially, and license their derivative works on different terms, provided the original work is properly cited and the use is non-commercial. See: http://creativecommons.org/ licenses/by-nc/4.0/

(c) Article author(s) (or their employer(s) unless otherwise stated in the text of the article) 2017. All rights reserved. No commercial use is permitted unless otherwise expressly granted.

\section{REFERENCES}

1 Owen N, Healy GN, Matthews CE, et al. Too much sitting: the population health science of sedentary behavior. Exerc Sport Sci Rev 2010;38:105-13.

2 Dogra S, Stathokostas L. Sedentary behavior and physical activity are independent predictors of successful aging in middle-aged and older adults. J Aging Res 2012;2012:1-8

3 Harvey JA, Chastin SF, Skelton DA. How sedentary are older people? A systematic review of the amount of sedentary behavior. J Aging Phys Act 2015;23:471-87.

4 Colley RC, Garriguet D, Janssen I, et al. Physical activity of canadian adults: accelerometer results from the 2007 to 2009 Canadian Health measures survey. Health Rep 2011:22:7-14.

5 Matthews CE, George SM, Moore SC, et al. Amount of time spent in sedentary behaviors and cause-specific mortality in US adults. Am J Clin Nutr 2012:95:437-45.

6 Sparling PB, Howard BJ, Dunstan DW, et al. Recommendations for physical activity in older adults. BMJ 2015;350:h100.

7 Van Cauwenberg J, Van Holle V, De Bourdeaudhuij I, et al. Diurnal patterns and correlates of older adults' Sedentary behavior. PLoS One 2015;10:e0133175.
8 Matthews $C E$, Chen $\mathrm{KY}$, Freedson PS, et al. Amount of time spent in sedentary behaviors in the United States, 2003-2004. Am J Epidemiol 2008;167:875-81.

9 Copeland JL, Clarke J, Dogra S. Objectively measured and self-reported sedentary time in older Canadians. Prev Med Rep 2015:2:90-5.

10 Diaz KM, Howard VJ, Hutto B, et al. Patterns of sedentary behavior in US Middle-Age and older adults: the REGARDS study. Med Sci Sports Exerc 2016:48:430-8.

11 Dogra S, Stathokostas L. Correlates of extended sitting time in older adults: an exploratory cross-sectional analysis of the Canadian Community Health Survey healthy aging cycle. Int J Public Health 2014;59:983-91.

12 O'Neill C, Dogra S. Different types of sedentary activities and their Association with perceived Health and Wellness among Middle-Aged and older adults: a CrossSectional analysis. Am J Health Promot 2016;30:150709150949009.

13 Arnardottir NY, Koster A, Van Domelen DR, et al. Objective measurements of daily physical activity patterns and sedentary behaviour in older adults: age, Gene/ Environment Susceptibility-Reykjavik Study. Age Ageing 2013:42:222-9.

14 Wilmot EG, Edwardson CL, Achana FA, et al. Sedentary time in adults and the association with diabetes, cardiovascular disease and death: systematic review and meta-analysis. Diabetologia 2012;55:2895-905.

15 Ford ES, Caspersen CJ. Sedentary behaviour and cardiovascular disease: a review of prospective studies. Int J Epidemio/ 2012;41:1338-53.

16 de Rezende LF, Rey-López JP, Matsudo VK, et al. Sedentary behavior and health outcomes among older adults: a systematic review. BMC Public Health 2014;14:333.

17 Hajduk AM, Chaudhry SI. Sedentary behavior and Cardiovascular risk in older adults: a scoping review. Curr Cardiovasc Risk Rep 2016;10:5.

18 Biswas A, Oh Pl, Faulkner GE, et al. Sedentary time and its association with risk for disease incidence, mortality, and hospitalization in adults: a systematic review and meta-analysis. Ann Intern Med 2015;162:123-32.

19 Ekelund U, Steene-Johannessen J, Brown WJ, et al. Does physical activity attenuate, or even eliminate, the detrimental association of sitting time with mortality? A harmonised meta-analysis of data from more than 1 million men and women. Lancet 2016;388:1302-10.

20 F Leask C, Sandlund M, A Skelton D, et al. Modifying older adults' Daily Sedentary Behaviour using an Asset-based solution: views from older adults. AIMS Public Health 2016:3:542-54.

21 Mcewan T, Tam-Seto L, Dogra S. Perceptions of Sedentary Behavior among socially Engaged Older adults. Gerontologist 2016:gnv689.

22 Wilkin LD, Haddock BL. Functional Fitness of Older adults. Act Adapt Aging 2011:35:197-209.

23 Fleg JL, Morrell CH, Bos AG, et al. Accelerated longitudinal decline of aerobic capacity in healthy older adults. Circulation 2005;112:674-82.

24 Rajan KB, Hebert LE, Scherr P, et al. Cognitive and physical functions as determinants of delayed age at onset and progression of disability. J Gerontol A Biol Sci Med Sci 2012:67:1419-26.

25 Welmer AK, Rizzuto D, Laukka EJ, et al. Cognitive and physical function in relation to the risk of Injurious Falls in Older adults: a Population-Based study. J Gerontol A Biol Sci Med Sci 2017;72:glw141.

26 Martinez-Martin P, Prieto-Flores M-E, Forjaz Maria Jo?o, et al. Components and determinants of quality of life in community-dwelling older adults. Eur J Ageing 2012:9:255-63.

27 United Nations DoEaSA, Population Division. World Population Ageing 2015, 2015.

28 Chastin SF, De Craemer M, Lien N, et al. The SOS-framework (Systems of sedentary behaviours): an international transdisciplinary consensus framework for the study of determinants, research priorities and policy on sedentary behaviour across the life course: a DEDIPAC-study. Int J Behav Nutr Phys Act 2016;13:83.

29 Chastin SF, Buck C, Freiberger E, et al. Systematic literature review of determinants of sedentary behaviour in older adults: a DEDIPAC study. Int I Behav Nutr Phys Act 2015;12:127.

30 Organization WH. Global Strategy and Action Plan on Ageing and Health. 2016

31 Standardized use of the terms "sedentary" and "sedentary behaviours". Appl Physiol Nutr Metab 2012:37.

32 Chastin SF, Schwarz U, Skelton DA, et al. Development of a consensus taxonomy of sedentary behaviors (SIT): report of Delphi Round 1. PLoS One 2013;8:e82313.

33 Hsu C-C, Sanford B. The Delphi Technique: making sense of Consensus Practical Assessment. Research \& Evaluation 2007;12:8

34 Jones J, Hunter D. Consensus methods for medical and health services research. BMJ 1995;311:376-80.

35 Day S, Mason R, Lagosky S, et al. Integrating and evaluating sex and gender in health research. Health Res Policy Syst 2016;14:75.

36 Gahagan J, Gray K, Whynacht A. Sex and gender matter in health research: addressing health inequities in health research reporting. Int J Equity Health 2015;14:12.

37 Heidari S, Babor TF, Castro P, et al. [Sex and gender equity in research: rationale for the SAGER guidelines and recommended use]. Epidemiol Serv Saude 2017;1:2.

38 Copeland J. Older adult's Sedentary Time: Measurement, Health Consequences and Interventions. BISM 2017.

39 Van Cauwenberg J, Van Holle V, De Bourdeaudhuij I, et al. Older adults' reporting of specific sedentary behaviors: validity and reliability. BMC Public Health 2014;14:734. 
40 Visser M, Koster A. Development of a questionnaire to assess sedentary time in older persons--a comparative study using accelerometry. BMC Geriatr 2013;13:80.

41 Gardiner PA, Clark BK, Healy GN, et al. Measuring older adults' sedentary time: reliability, validity, and responsiveness. Med Sci Sports Exerc 2011;43:2127-33.

42 Gennuso KP, Matthews CE, Colbert LH. Reliability and validity of 2 Self-Report measures to assess sedentary behavior in older adults. J Phys Act Health 2015;12:727-32.

43 Bastone AC, Moreira BS, Vieira RA, et al. Validation of the human activity profile questionnaire as a measure of physical activity levels in older community-dwelling women. J Aging Phys Act 2014;22:348-56.

44 Jefferis BJ, Sartini C, Ash S, et al. Validity of questionnaire-based assessment of sedentary behaviour and physical activity in a population-based cohort of older men; comparisons with objectively measured physical activity data. Int J Behav Nutr Phys Act 2016;13:14.

45 España-Romero V, Golubic R, Martin KR, et al. Comparison of the EPIC Physical Activity Questionnaire with combined heart rate and movement sensing in a nationally representative sample of older british adults. PLoS One 2014;9:e87085.

46 Aguilar-Farías N, Brown WJ, Olds TS, et al. Validity of self-report methods for measuring sedentary behaviour in older adults. J Sci Med Sport 2015;18:662-6.

47 Clark BK, Lynch BM, Winkler EA, et al. Validity of a multi-context sitting questionnaire across demographically diverse population groups: ausdiab3. Int J Behav Nutr Phys Act 2015;12:148.

48 Pavey TG, Peeters GG, Brown WJ. Sitting-time and 9-year all-cause mortality in older women. Br J Sports Med 2015;49:95-9.

49 Matthews CE, Moore SC, Sampson J, et al. Mortality benefits for replacing sitting time with different physical activities. Med Sci Sports Exerc 2015;47:1833-40.

50 Stamatakis E, Davis M, Stathi A, et al. Associations between multiple indicators of objectively-measured and self-reported sedentary behaviour and cardiometabolic risk in older adults. Prev Med 2012;54:82-7.

51 Smith L, Hamer M. Television viewing time and risk of incident diabetes mellitus: the English Longitudinal Study of Ageing. Diabet Med 2014;31:1572-6.

52 Gardiner PA, Healy GN, Eakin EG, et al. Associations between television viewing time and overall sitting time with the metabolic syndrome in older men and women: the australian Diabetes, Obesity and Lifestyle study. J Am Geriatr Soc 2011;59:788-96.

53 Hallal PC, Andersen LB, Bull FC, et al. Global physical activity levels: surveillance progress, pitfalls, and prospects. Lancet 2012;380:247-57.

54 English KL, Paddon-Jones D. Protecting muscle mass and function in older adults during bed rest. Curr Opin Clin Nutr Metab Care 2010;13:34-9.

55 Dunlop DD, Song J, Arnston EK, et al. Sedentary time in US older adults associated with disability in activities of daily living independent of physical activity. J Phys Act Health 2015;12:93-101.

56 Chastin SF, Ferriolli E, Stephens NA, et al. Relationship between sedentary behaviour, physical activity, muscle quality and body composition in healthy older adults. Age Ageing 2012;41:111-4.

57 Gautam R, Saito T, Kai I. Leisure and religious activity participation and mental health: gender analysis of older adults in Nepal. BMC Public Health 2007;7:299.
58 Fitzsimons CF, Kirk A, Baker G, et al. Using an individualised consultation and activPAL ${ }^{T M}$ feedback to reduce sedentary time in older scottish adults: results of a feasibility and pilot study. Prev Med 2013;57:718-20.

59 Lewis LK, Rowlands AV, Gardiner PA, et al. Small steps: preliminary effectiveness and feasibility of an incremental goal-setting intervention to reduce sitting time in older adults. Maturitas 2016;85:64-70.

60 Gardiner PA, Eakin EG, Healy GN, et al. Feasibility of reducing older adults' sedentary time. Am J Prev Med 2011;41:174-7.

61 Matei R, Thuné-Boyle I, Hamer M, et al. Acceptability of a theory-based sedentary behaviour reduction intervention for older adults ('On Your Feet to Earn Your Seat'). BMC Public Health 2015;15:606.

62 Rosenberg DE, Bellettiere J, Gardiner PA, et al. Independent associations between sedentary behaviors and mental, cognitive, physical, and functional Health among Older adults in Retirement Communities. J Gerontol A Biol Sci Med SCi 2016;71:78-83.

63 Barone Gibbs B, Brach JS, Byard T, et al. Reducing Sedentary Behavior Versus increasing Moderate-to-Vigorous intensity Physical activity in Older adults: a 12-Week Randomized, Clinical trial. J Aging Health 2016.

64 Asaoka S, Fukuda K, Tsutsui Y, et al. Does television viewing cause delayed and/or irregular sleep?wake patterns? Sleep Biol Rhythms 2007:5:23-7.

65 Chang AK, Fritschi C, Kim MJ. Sedentary behavior, physical activity, and psychological health of korean older adults with hypertension: effect of an empowerment intervention. Res Gerontol Nurs 2013:6:81-8.

66 Sjögren P, Fisher R, Kallings L, et al. Stand up for health--avoiding sedentary behaviour might lengthen your telomeres: secondary outcomes from a physical activity RCT in older people. Br J Sports Med 2014;48:1407-9.

67 Kallings LV, Sierra Johnson J, Fisher RM, et al. Beneficial effects of individualized physical activity on prescription on body composition and cardiometabolic risk factors: results from a randomized controlled trial. Eur J Cardiovasc Prev Rehabil 2009;16:80-4

68 Lee RE, King AC. Discretionary time among older adults: how do physical activity promotion interventions affect sedentary and active behaviors? Ann Behav Med 2003;25:112-9.

69 Fanning J, Porter G, Awick EA, et al. Effects of a DVD-delivered exercise program on patterns of sedentary behavior in older adults: a randomized controlled trial. Prev Med Rep 2016;3:238-43.

70 Burke L, Lee AH, Jancey J, et al. Physical activity and nutrition behavioural outcomes of a home-based intervention program for seniors: a randomized controlled trial. Int J Behav Nutr Phys Act 2013;10:14

71 LeBlanc AG, Berry T, Deshpande S, et al. Knowledge and awareness of canadian physical activity and sedentary Behaviour guidelines: a synthesis of existing evidence. Appl Physiol Nutr Metab 2015;40:716-24.

72 United Kingdom Government DoH. Physical activity guidelines for older adults (65+ years, 2011.

73 Buckley JP, Hedge A, Yates T, et al. The sedentary office: a growing case for change towards better health and productivity. expert statement commissioned by Public Health England and the active Working Community Interest Company. British Journal of Sports Medicine 2015. 